\title{
Enabling Self-healing Smart Grid Through Jamming Resilient Local Controller Switching
}

\author{
Hongbo Liu, Yingying Chen, Mooi Choo Chuah, IEEE Fellow, Jie Yang, and H. Vincent Poor, IEEE Fellow
}

\begin{abstract}
A key component of a smart grid is its ability to collect useful information from a power grid for enabling control centers to estimate the current states of the power grid. Such information can be delivered to the control centers via wireless or wired networks. It is envisioned that wireless technology will be widely used for local-area communication subsystems in the smart grid (e.g., in distribution networks). However, various attacks with serious impact can be launched in wireless networks such as channel jamming attacks and denial-of-service attacks. In particular, jamming attacks can cause significant damages to power grids, e.g., delayed delivery of time-critical messages can prevent control centers from properly controlling the outputs of generators to match load demands. In this paper, a communication subsystem with enhanced self-healing capability in the presence of jamming is designed via intelligent local controller switching while integrating a retransmission mechanism. The proposed framework allows sufficient readings from smart meters to be continuously collected by various local controllers to estimate the states of a power grid under various attack scenarios. The jamming probability is also analyzed considering the impact of jammer power and shadowing effects. In addition, guidelines on optimal placement of local controllers to ensure effective switching of smart meters under jamming are provided. Via theoretical, experimental and simulation studies, it is demonstrated that our proposed system is effective in maintaining communications between smart meters and local controllers even when multiple jammers are present in the network.
\end{abstract}

Index Terms—Smart Grid, Local controller switching, Jamming.

\section{INTRODUCTION}

$\mathrm{S}^{\mathrm{s}}$ MART grid is proposed to improve the efficiency and reliability of existing power grids by adding automated monitoring, communication, self-diagnosis, and demand-response capabilities. Technically, the smart grid [1] can be divided into smart infrastructure, smart management, and smart protection systems. The smart infrastructure which supports bidirectional flow of electricity and information is further subdivided into smart energy, information, and communication subsystems [1]. The smart energy subsystem takes care of advanced electricity generation and delivery, whereas the smart information subsystem involves advanced metering, monitoring and management. The smart communication subsystem facilitates information exchanges among systems, devices, and applications.

We focus on the smart communication subsystem that is used to support the smart information subsystem for distribution networks. Wireless technology is promising for this application as it is relatively easy to install, and also supports high-rate data transmissions, e.g., up to $100 \mathrm{Mbps}$ in a range of $50 \mathrm{~km}$ with the IEEE 802.16 protocol [2]. Hence it is expected that the last mile of the communication subsystem, e.g., the communication between smart meters and controllers, will often be wireless in nature. Such

- Hongbo Liu is with the Department of Computer Information and Graphics Technology, Indiana University Purdue University at Indianapolis, IN 46202.

E-mail: h145@iupui.edu

- Yingying Chen is with the Department of Electrical and Computer Engineering, Stevens Institute Technology, Hoboken, NJ 07330. E-mail: yingying.chen@ stevens.edu

- Mooi Choo Chuah is with the Department of Computer Science and Engineering, Lehigh University, Bethlehem, PA 18015. E-mail: chuah@cse.lehigh.edu

- Jie Yang is with the Department of Computer Science, Florida State University, Tallahassee, FL 32306.

E-mail: jyang5@fsu.edu

- H. Vincent Poor is with the Department of Electrical Engineering, Princeton University, Princeton, NJ 08544.

E-mail: poor@princeton.edu

Manuscript received $X X X X$; revised $X X X X$. a highly distributed wireless system in the smart grid makes it more vulnerable to various adversary attacks [3], [4]. In particular, jamming attacks aim to disrupt the data communication between smart meters and local controllers, which is considered as an important first step in an adversary's attempt to launch a variety of attacks. For instance, an adversary can delay or block smart meter reading collection and jam real-time price signals transmitted in the last mile to undermine the demand-respond system [5]. Even small-scale jamming attacks in local area networks can cause partial unavailability of data samples for state estimation [6], [7]. Furthermore, an attacker can launch a malicious jamming attack which prevents a substation from collecting complete data, and also simultaneously launch a false data injection attack to provide fabricated data to the substation. Such combined attacks can cause the substation to use the corrupted information for state estimation and result in producing the wrong control actions, causing dire consequences on the smart grid operations.

Compared to the legacy power systems, the smart grid operates in a more open communication network covering large geographical areas. Due to the critical importance of power infrastructures, resilience operation in communication networks is essential to sustain network availability. Given the large geographical coverage of the smart grid, eliminating jammers manually by dispatching technicians is resource consuming and less practical. The smart grid needs to have enhanced self-healing capability to maintain normal network operations in the presence of attacks. Thus, coping with jamming serves as the first line of defense to achieve reliable, secure, and real-time data delivery and customer management in the smart grid. Adopting traditional channel hopping techniques [8], [9] in smart meters and local controllers is useful in alleviating jamming effects. However, smart attackers may adjust their jamming strategies based on the observations they gather from the on-going communications between smart meters and controllers. For example, a jammer with fast hopping speed can quickly identify the channel in use between smart meters and a 
local controller, making the employment of pure channel hopping less effective. Therefore, more intelligent defense strategies need to be devised.

Our basic idea is to exploit all the available channels between smart meters and controllers that can be used to communicate and maintain high data delivery rate under jamming. In this paper, we propose a framework that enables smart meters to identify nearby local controllers in addition to its primary local controller. It allows smart meters and local controllers to determine appropriate channels to communicate with one another when jamming is present. Our framework provides enhanced flexibility, which allows smart meters to communicate with any nearby controllers that they can hear on any available channel, and hence increases the successful data delivery rate in the distribution network under jamming attacks. Through theoretical analysis, experimental study and simulation evaluation, we show that our framework is effective in allowing smart meters and controllers to continue their communications even under malicious attacks when multiple and colluded jammers are employed. Our work confirms the feasibility of effectively coping with jamming using intelligent local controller switching in the smart communication subsystem and is the first step towards providing the self-healing feature in a smart grid under adversarial conditions. Our main contributions in this paper are summarized as follows:

- We propose a framework that exploits intelligent controller switching together with channel hopping to provide resilience of data delivery under jamming in a distribution network.

- We develop a retransmission scheme integrated with the proposed framework to further ensure successful data delivery from smart meters.

- We perform a theoretical analysis of jamming probability based on the impact of jammer transmission power and shadowing effects.

- We build a testbed using Micaz motes implementing the proposed intelligent controller switching strategy to show the feasibility of such a framework.

- We conduct large-scale performance evaluations of our framework with multiple independent and colluded jammers using simulation studies.

- We analyze the optimal placement of local controllers to ensure effective switching of smart meters under jamming.

The rest of the paper is organized as follows. We put our work in the broader context in Section 2. In Section 3, we describe the smart grid network architecture and the attack model adopted in this work. We then present our proposed framework enabling intelligent local controller switching in Section 4. Next, we provide the theoretical analysis of our proposed strategy in Section 5. We describe the testbed implementation of local controller switching with channel hopping and our experimental result in Section 6. The extensive performance evaluation is conducted through simulation in Section 7. In Section 8, we analyze the optimal coverage of local controller placement that supports intelligent local controller switching. Finally, we conclude our work in Section 9.

\section{Related Work}

Jamming attacks are serious security threats disrupting reliability of wireless communication, and have been extensively studied in wireless networks [8], [10]-[13]. For example, jamming attack detection was studied by Liu et al. [10], [13] in the context of commodity wireless devices and wireless sensor networks. Besides jamming attack detection, spread spectrum techniques including both Frequency Hopping (FH) and Direct Sequence Spread Spectrum (DSSS) have been widely used to defend against jamming attacks in wireless communications [9], [14], [15] at the expense of advanced transceivers. In particular, for FrequencyHopping Spread Spectrum (FHSS) [9], a transmitter and receiver synchronously switch among many different frequency channels following a common pseudo-random hopping sequence known to each other. If the number of frequency channels is large enough, it will greatly increase the cost of jamming attacks, since the jammer either needs to know the pseudo-random hopping sequence or should be able to mount the jamming attack across a wide frequency band. For DSSS [14], [15], the receiver multiplies each data bit with a Pseudo-Noise (PN) digital signal which is transmitted at a higher rate than the data, consequently the data will be spread over a wider frequency bandwidth. This makes the legitimate signal hard to detect by attackers and also allows for easier bit recovery by providing bit level error correction. The two spread spectrum techniques above do not eliminate jamming but force attackers to spend more energy to mount an equivalent attack. Furthermore, several uncoordinated frequency hopping (UFH) schemes have been proposed to enable jamming-resistant communication in the presence of jamming attacks without a pre-shared secret [12], [16]-[18]. Particularly, the shared secret between transmitter and receiver is established via the Uncoordinated Seed Disclosure in Frequency Hopping under the presence of jamming. Besides FHSS and DSSS, other defense strategies include the use of error correcting codes [19] to increase the likelihood of decoding corrupted packets, spatial retreats [20] to move out of jammed regions geographically, anti-jamming timing channels [21], wormhole-based anti-jamming techniques [22], Multi-Channel Ratio (MCR) Decoding [23], and localizing jammers for physically neutralizing the jamming attacks [24][26].

Recently, a few works have been focused on studying jamming attacks in the context of smart grid applications. Li et al. discussed Denial-of-Service (DoS) jamming of wireless communication in the smart grid and studied the possibility of manipulating the power market by jamming the pricing signal [5], [27]. Lu et al. provided a study on the impact of jamming attacks against time-critical network applications (e.g. power grids), and observed that generating a fair amount of camouflage traffic in the network could minimize the message delay for the smart grid applications under jamming attacks [4], [28]. Su et al. studied the anti-jamming problem in a multi-radio multi-channel multihop (M3) network for supporting the smart grid from a cross-layer perspective, and proposed a dynamic channel assignment algorithm based on the analysis of the capacity of the victimized links via machine learning algorithms [29]. Unlike the previous work, we focus on designing a self-healing communication subsystem with local controller switching that is robust against jamming attacks. Our work is novel in that we exploit all the available channels between smart meters and controllers to increase the data delivery rate under jamming.

\section{System OVerview}

\subsection{Smart Grid Network Architecture}

In this work, we adopt the smart grid architecture described in [1] which consists of three major systems, namely smart infrastructure, smart management and smart protection systems. 


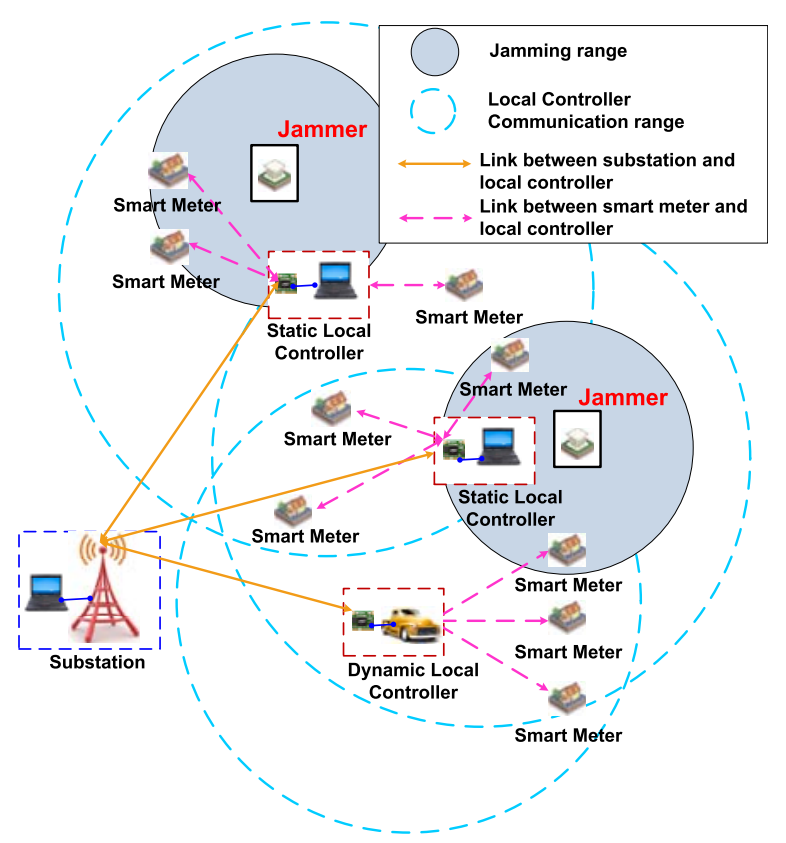

Fig. 1. Architecture of the smart grid distribution network and illustration of jammer deployment.

We focus on the smart communication subsystem which supports the smart information subsystem within the smart infrastructure system for distribution networks as shown in Figure 1. Typically, such a communication subsystem is hierarchical in nature with devices within each geographical region forming different subnetworks. A typical smart grid communication subsystem consists of one or more substations, with each substation supervising the operations of multiple local controllers in a particular region. The substation is responsible for the information aggregation from all the local controllers. Each local controller interacts with multiple smart meters for supporting power consumption reading collection, operation data management, and data acquisition control. The smart meters within a geographical region communicate with a local controller via ZigBee-based radios while the local controllers communicate with one another via wireless mesh network. Furthermore, the local controllers communicate with the substation controller via power line communications or cellular networks. Thus, the smart grid communication subsystem comprises the ZigBee networks, the wireless mesh networks and the cellular networks

We assume that the smart grid communication subsystem is designed such that any smart meter can communicate with several local controllers, but it has only one primary local controller to which it delivers power consumption readings during normal operations. Smart meters do not communicate with one another. Under normal operations, a local controller broadcasts beacons in a particular channel and smart meters scan all channels to find nearby local controllers to associate with.

\subsection{Attack Model}

The shared nature of the wireless medium creates opportunities for adversaries employing jammers to disrupt data delivery between smart meters and local controllers in the smart grid, from delayed delivery of time-critical messages to complete denialof-service [3], [30]. As the network has multiple channels, the jammer can adopt a wide range of strategies to disrupt message delivery. The attacker possesses the knowledge of the available channels between a local controller and smart meters under its coverage. Thus, a jammer could target a particular local controller to disrupt its communication. Furthermore, we assume that a jammer can only disturb the message communication in one channel at each time slot.

We consider two major jamming types: random and reactive. A random jammer randomly selects a channel used between a local controller and smart meters at each time slot and disrupts the data communication without monitoring the channel activities, while a reactive jammer monitors a channel and only launches the attack when there are activities on the channel.

In addition, we consider both single and multiple stationary jammers. With multiple jammers, we further consider independent versus colluded jammers. With multiple independent jammers, the communications between smart meters and local controllers in multiple channels could be disrupted at each time slot. Multiple colluded jammers can collaboratively launch an attack targeting a particular channel at a time slot, causing severe channel interference.

\section{Framework of Intelligent local Con- tROLLeR SWITCHING WITH ChanNel HOPPING (LCS-CH)}

Previous studies mainly rely on channel hopping techniques [8], [9], [12], [17] to mitigate jamming attacks in wireless networks. The basic idea of the channel hopping technique is: the communication between the sender and receiver at any particular time slot takes place using a particular channel chosen from a sequence of pre-defined channels (referred to as a hopping sequence), which are pre-loaded into communication devices. Typically communications between smart meters and local controllers are based on 802.15.4-equivalent radios which only have a fixed number of available channels. For a large deployment scenario where we need to consider having multiple local controllers operating on independent channels, each local controller can only be assigned a limited number of channels. Thus, despite the recent success of employing channel hopping techniques to achieve jamming resilient wireless communication, limited channel resources available on each local controller make the channel hopping technique insufficient to defend against jamming attacks in a smart grid. The jammers with fast hopping speed would make a pure channel hopping scheme less effective, since the jammer can quickly find the channel in use between the local controller and smart meters. Therefore, we propose a framework that actively performs local controller switching with channel hopping to thwart jamming attacks. With our proposed framework, a smart meter can utilize all available channels from nearby local controllers to send its readings, and hence increase the chances of such readings being successfully collected by one of the nearby local controllers under jamming, and subsequently by the substation.

\subsection{Framework Design}

In this work, we focus on alleviating jamming effects on smart meters and local controllers after an attack is detected. Thus, we assume that the network is able to detect the presence of jammers using existing techniques [8], [10]. For instance, the interference from jammers degrades the signal-to-noise ratio (SNR) of any received packet from a smart meter, the packet may not be decodable at the corresponding local controller. When a consecutive sequence of packets are undecodable, the network concludes that 


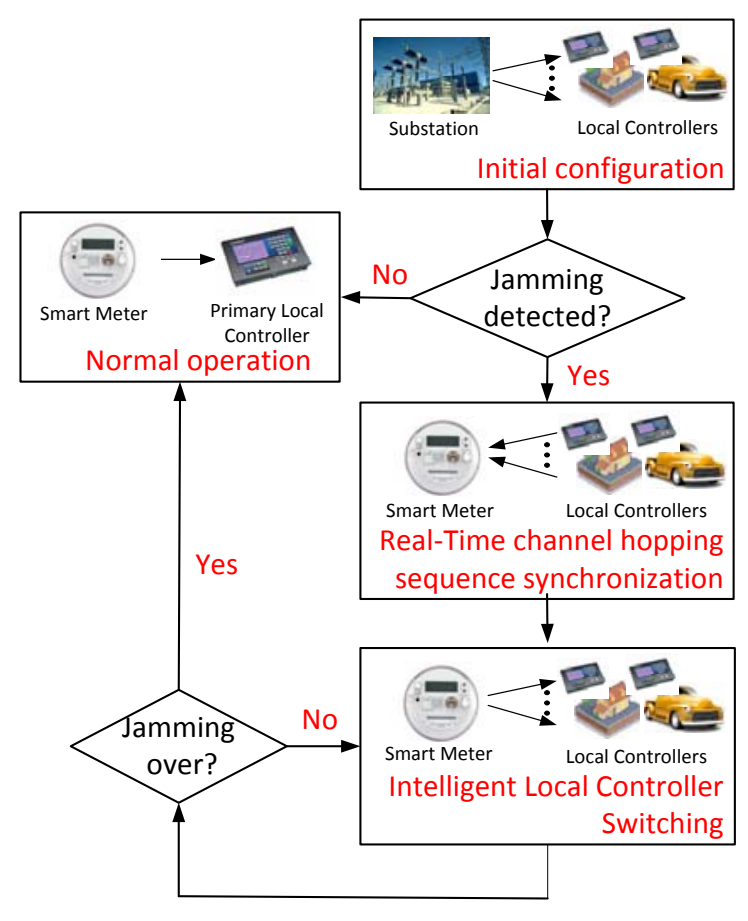

Fig. 2. Framework overview.

there is a jammer present. We propose a framework such that each smart meter is associated with a primary local controller and can also communicate with a set of nearby local controllers. Each local controller is pre-configured with a number of channel hopping sequences. The length of each channel hopping sequence is the same for all local controllers. The channel used in any particular time slot within a hopping sequence of a particular local controller does not overlap with any nearby local controllers. The channel hopping technique is triggered by the affected local controllers after a jamming attack is detected.

We assume that this communication subsystem runs as a timeslotted system, i.e. at each time slot, the local controller can decide which frequency channel it will use to communicate with smart meters that are associated with it. Our framework contains three main aspects: initial configuration in the smart grid, real-time channel hopping sequence synchronization between smart meters and the local controller under jamming, and intelligent local controller switching to alleviate jamming and increase successful data delivery rate.

Initial Configuration. All the channel hopping sequences are generated and distributed by the substation, which manages a set of local controllers. In our framework, we consider a hybrid deployment of static and dynamic local controllers. In particular, static local controllers are permanently placed by a utility company, while dynamic local controllers could be utility trucks driving around to collect data from smart meters. During the deployment of a static local controller, it is uploaded with a number of channel hopping sequences, which ensures that nearby local controllers have no collision with each other on channel hopping. The dynamic local controllers are also pre-configured with multiple channel hopping sequences.

Real-Time Channel Hopping Sequence Synchronization. When jamming is detected by the network by employing existing techniques [8], [10], smart meters and local controllers need to synchronize with each other to perform channel hopping. The af- fected local controllers (including both static and dynamic) utilize the one-time pseudo-random hopping pattern technique [12] to send out new beacons. Each new beacon message includes the channel hopping sequence, selected from the pre-configured set of channel hopping sequences, and the corresponding starting time of channel hopping. Such beacons are transmitted multiple times, each using a different pseudo-random hopping pattern, to ensure the information can be received by all the relevant smart meters.

Intelligent Local Controller Switching. Since smart meters have the opportunity to find more than one available local controllers in our framework, they can choose to switch to the appropriate nearby local controllers once they receive the channel hopping sequences from them. In our framework, each smart meter can actively decide which nearby local controller to connect to at each time slot, and hence increase the successful data delivery rate under jamming. In case no overlapping local controller is available for a particular smart meter, then only frequency hopping technique will be employed.

\subsection{Collision-Free Channel Hopping Sequence Distri- bution}

To defend against the jamming attack via the channel hopping technique, the substation constructs and distributes a set of channel hopping sequences to each local controller. The predefined hopping sequences among nearby local controllers should follow the collision-free principle, where any two channel hopping sequences have no interference with each other. The technique for constructing collision-free channel hopping sequences can be based on finite field theory from existing work [9]. To illustrate the collision-free channel hopping sequence distribution, we use an example when each local controller is assigned with only one channel hopping sequence. Assume 4 local controllers are deployed in the area of interest. There are a total of 20 available channels. Each local controller has one hopping sequence containing 5 channels for communicating with smart meters. The channel hopping pattern for these 4 local controllers can then be designed as follows:

$$
\begin{aligned}
& L C_{1} \\
& L C_{2} \\
& L C_{3} \\
& L C_{4}
\end{aligned}\left[\begin{array}{ccccc}
1 & 5 & 9 & 13 & 17 \\
2 & 6 & 10 & 14 & 18 \\
3 & 7 & 11 & 15 & 19 \\
4 & 8 & 12 & 16 & 20
\end{array}\right]
$$

where each row corresponds to the channel hopping sequence of one particular local controller $L C_{i}$ with $i=1, \cdots, 4$ at different time slots; each column corresponds to the channels for 4 local controllers at one particular time slot $t_{j}$ with $j=1, \cdots, 5$.

When a jamming attack is detected, each affected local controller chooses from its pre-configured collision-free channel hopping sequence and starts sending out beacons by following a one-time pseudo-random hopping pattern [12]. The beacon message contains the local controller's identifier, the selected channel hopping sequence, and the starting time for channel hopping. The beacon message is transmitted multiple times by following different pseudo-random hopping patterns. Each transmission is independent of each other. Each affected smart meter randomly hops through all channels, and eventually it will have an overlapping channel with a local controller and receive the disclosed channel hopping sequence. Since each smart meter can communicate with several nearby local controllers, it is possible that the smart meter can receive the channel hopping sequence from multiple local controllers. However, merely using the channel hopping technique is not sufficient to maintain high data delivery 
rate under jamming as a jammer may follow the same procedure as smart meters to learn the channel hopping sequences in the affected area.

\subsection{Intelligent Local Controller Switching with Channel Hopping (LCS-CH)}

Our objective is to make use of all the available channels from nearby local controllers so as to maintain regular data delivery under jamming. To achieve this goal, we leverage the collaborative efforts from a smart meter's nearby local controllers. Instead of relying on the pure channel hopping technique, which has limited capability on defending against jamming attacks, we propose active local controller switching on top of channel hopping to increase successful data delivery rate.

We next describe how a smart meter comes up with a strategy to perform active local controller switching under jamming. Let us denote the channel hopping sequence $F_{i}$ of the local controller $L C_{i}$ as a $k$-length vector:

$$
F_{i}=\left[f_{i, 1}, f_{i, 2}, \cdots, f_{i, j}, \cdots, f_{i, k}\right]
$$

where $f_{i, j}$ corresponds to a particular channel in the frequency hopping sequence at $j$ th time slot with $1 \leq j \leq k$. Considering all neighboring local controllers with collision-free channel hopping sequences, the smart meter defines its channel selection matrix as:

$$
F_{I \times k}=\left[\begin{array}{ccccc}
f_{1,1} & f_{1,2} & \cdots & f_{1, k-1} & f_{1, k} \\
f_{2,1} & f_{2,2} & \cdots & f_{2, k-1} & f_{2, k} \\
& \cdots & & \cdots & \\
f_{I, 1} & f_{I, 2} & \cdots & f_{I, k-1} & f_{I, k}
\end{array}\right],
$$

where each row corresponds to the selected channel hopping sequence for one nearby local controller and again $f_{i, j}$ represents the channel at $j$ th time slot of a neighboring local controller $L C_{i}$. The smart meter constructs $F_{I \times k}$ after real-time channel hopping sequence synchronization.

The smart meter then constructs the controller switching matrix $U_{I \times k}$ based on the channel hopping sequence received from nearby local controllers:

$$
U_{I \times k}=\left[u_{1}, \cdots, u_{j}, \cdots, u_{k}\right],
$$

where $u_{j}$ represents a $I$-length column vector that has only one non-zero entry with $u_{j}^{T} u_{j}=1$ and $j=1, \cdots, k$ time slots. It represents which local controller is selected at $j$ th time slot during channel hopping. Furthermore, $u_{j}(i)=1$ indicates that the smart meter chooses $i$ th local controller at $j$ th time slot with $1 \leq j \leq k$. For instance, $u_{2}=[0,0,0,1,0]$ means the smart meter choose the fourth local controller at the second time slot.

Integrating the channel selection and controller switching matrices, the smart meter can then derive its channel hopping strategy as follows:

$$
S_{1 \times k}=1_{1 \times I}\left(F_{I \times k} \odot U_{I \times k}\right)
$$

where $\odot$ represents element-wise product. Such a strategy ensures the smart meter finds an available channel to deliver data at any time slot under jamming. Although the jammers may have the capability to learn all the selected channel hopping sequences by eavesdropping in the affected area, jammers do not have the ability to jam all the channels at the same time. Figure 3 illustrates our intelligent local controller switching scheme. When only channel hopping is used as shown in Figure 3 (a), a smart meter hops among multiple channels of the primary local controllers. When there are multiple local controllers nearby, a smart meter can
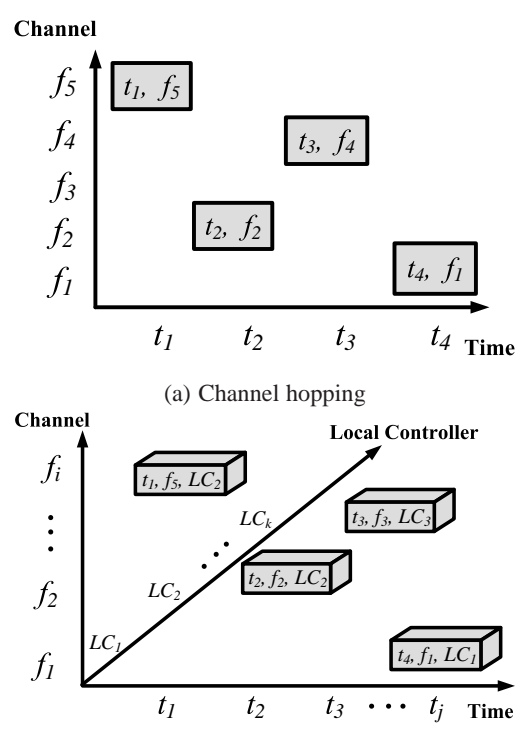

(b) LCS-CH

Fig. 3. Illustration of intelligent local controller switching scheme.

switch among these local controllers for data delivery. Using active local controller switching with channel hopping, a smart meter can take advantages of all available channels from different nearby local controllers as shown in Figure 3 (b).

\subsection{Retransmission Scheme}

After SMs send their packets to a particular LC, the LC needs to acknowledge to these SMs whether their packets have been successfully received. Otherwise, if some packets from the SMs are lost due to jamming, the relevant information from smart meters would be missed by the control center. To minimize such losses, we design a retransmission scheme between any LC and SMs in our proposed framework.

The basic idea is that a particular LC reserves several time slots to inform the SMs under its coverage of any successfully received packets, and in subsequent time slots relevant SMs will re-transmit packets that are not received. Each SM selects its retransmission slot based on its unique identifier. The information flow of the retransmission mechanism is shown in Figure 4:

Step 1. Each packet transmitted by a SM is given a unique sequence number, and marked with the identifier (ID) of that SM. Then, the packet is sent to the particular LC at different time slots which is defined in the local controller switching matrix shown in Equation 2. Note that each local controller switching matrix spans $T$ time slots.

Step 2. The LC collects the packets from multiple SMs in successive $m T$ time slots, and saves the sequence numbers and the corresponding IDs of SMs contained in these successfully received packets. After several rounds of packet transmission lasting for $m T$ time slots, the next $T$ time slots, which are shown as $(m+1) T$ in Figure 4 , are reserved for LC to acknowledge SMs on which packets are successfully received. The Ack packet, which is broadcasted at every $(m+1) T$ time slots, contains all the received packet sequence numbers and their corresponding SMs' IDs. This Ack packet is sent repeatedly several times over different channels as chosen according to Equation 2. We assume that the information received in these $(m+1) T$ time slots will be useful for the decision process at the control center. Such repeated 


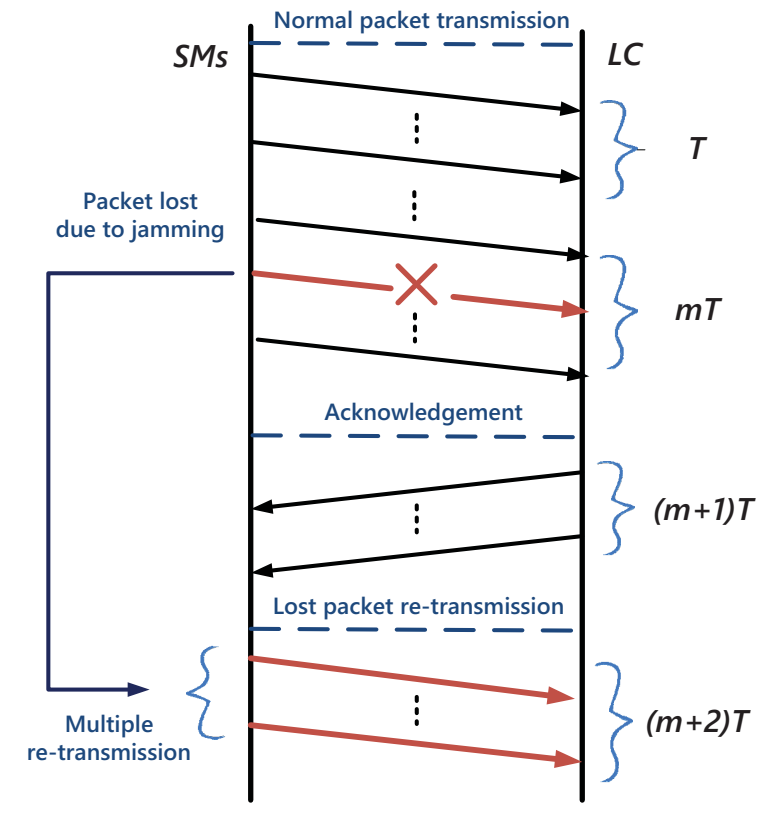

Fig. 4. Retransmission mechanism.

transmissions serve two purposes: (i) they reduce the probability of the ACK packet being jammed; (ii) since SMs received Acks from multiple nearby LCs, the SMs and LCs may not always be in the same channel. Multiple transmissions of the Ack packet can increase the chance of packet reception by the SMs even if they are not on the same channel as the corresponding LC at certain time slots.

Step 3. Once the SMs receive the acknowledgment packets from a LC, the lost packets, whose sequence numbers do not appear in the Ack, will be transmitted again. If any SM does not receive the Ack packet, it will just wait until another round of reserved time slots to receive later acknowledgment from that LC, and perform the retransmission accordingly.

Through the above steps, both LCs and SMs keep track of the lost packets under jamming and retransmit them again so that the data loss from SMs will be minimized.

\section{Analysis of Local Controller SWITCHing With Channel Hopping (LCS-CH)}

\subsection{Jamming Probability of Local Controller Switching With Channel Hopping (LCS-CH)}

In this section, we derive the probability that a smart meter cannot deliver its data to a local controller under jamming. We refer such a probability as jamming probability. We compare the jamming probability when using merely channel hopping technique to applying local controller switching with channel hopping (LCS$\mathrm{CH})$ after the jamming attack is detected.

Under jamming, the received power at a local controller is from both the smart meter it communicates with $\left(P_{L C_{i}, S M_{j}}\right)$ and the jammer $\left(P_{L C_{i}, J}\right)$. We use a single jammer as an example and describe the received power at local controller $L C_{i}$ using a logdistance path loss propagation model:

$$
\begin{aligned}
& P_{L C_{i}, S M_{j}}=P_{T}-P L_{0}-10 \gamma \log _{10}\left(\frac{d_{L C_{i}, S M_{j}}}{d_{0}}\right)-X_{g} \\
& P_{L C_{i}, J}=P_{J}-P L_{0}-10 \gamma \log _{10}\left(\frac{d_{L C_{i}, J}}{d_{0}}\right)-X_{g},
\end{aligned}
$$

where $P_{T}$ and $P_{J}$ represent the transmission power of the smart meter and the jammer. $X_{g}$ is a Gaussian random variable with distribution $N\left(0, \sigma^{2}\right)$, reflecting the attenuation caused by flat fading. $d_{L C_{i}, J}$ and $d_{L C_{i}, S M_{j}}$ are the distances from smart meter and jammer to local controller respectively.

When the communication between the local controller $L C_{i}$ and the smart meter $S M_{j}$ on the channel $f_{k}$ is disrupted by the jammer, the signal-to-noise ratio (at the local controller $L C_{i}$ from smart meter $\left.S M_{j}\right) S N R_{L C_{i}, S M_{j}}^{k}$ is less than a threshold $\gamma_{0}$. This signal-to-noise ratio can be represented as:

$$
\begin{aligned}
S N R_{L C_{i}, S M_{j}}^{k} & =P_{L C_{i}, S M_{j}}-P_{L C_{i}, J} \sim N\left(\mu, 2 \sigma^{2}\right) \\
& \sim N\left(P_{T}-P_{J}-10 \gamma \log _{10}\left(\frac{d_{L C_{i}, S M_{j}}}{d_{L C_{i}, J}}\right), 2 \sigma^{2}\right) .
\end{aligned}
$$

Then the possibility that a jammer successfully disrupts the communication between $S M_{j}$ and $L C_{i}$ on channel $f_{k}$ depends on the propagation model. And the jamming probability can be represented as:

$$
\operatorname{Prob}\left(S N R_{L C_{i}, S M_{j}}^{k}<\gamma_{0}\right)=\int_{-\infty}^{\gamma_{0}} \frac{1}{2 \sigma \sqrt{\pi}} e^{-\frac{\left(S N R_{L C_{i}, S M_{j}}^{k}-\mu\right)^{2}}{4 \sigma^{2}}} .
$$

When only the traditional frequency hopping technique is used under jamming, $S M_{j}$ can communicate with its primary local controller $L C_{i}$ through a set of independent channels from the selected channel hopping sequence. The jamming probability $\operatorname{Prob}\left(S M_{j}\right)^{C H}$ between $L C_{i}$ and $S M_{j}$ at time slot $t$ can then be derived as:

$$
\begin{aligned}
& \operatorname{Prob}\left(S M_{j}\right)^{C H} \\
= & \operatorname{Prob}\left(f^{J}(t)=f_{k} \& f^{S M_{j}}(t)=f_{k} \mid S N R_{L C_{i}, S M_{j}}^{k}<\gamma_{0}\right) \\
& \times \operatorname{Prob}\left(S N R_{L C_{i}, S M_{j}}^{k}<\gamma_{0}\right) \\
= & \operatorname{Prob}\left(f^{J}(t)=f_{k}\right) \operatorname{Prob}\left(f^{S M_{j}}(t)=f_{k}\right) \\
& \times \operatorname{Prob}\left(S N R_{L C_{i}, S M_{j}}^{k}<\gamma_{0}\right) \\
= & \frac{1}{N_{i} \times n} \int_{-\infty}^{\gamma_{0}} \frac{1}{2 \sigma \sqrt{\pi}} e^{-\frac{\left(S N R_{L C_{i}, S M_{j}}^{k}-\mu\right)^{2}}{4 \sigma^{2}}},
\end{aligned}
$$

where $f^{J}(t)$ and $f^{S M_{j}}(t)$ represent the channels used by the jammer and smart meter $S M_{j}$ at time slot $t . n$ indicates the number of channels that the jammer tries to disrupt, and $N_{i}$ is the total number of channels in the selected hopping sequence on $L C_{i} . f_{k}$ is one of the available channels on single local controller.

When our proposed LCS-CH framework is applied, the smart meter $S M_{j}$ actively perform local controller switching. Assume there are $I$ nearby local controllers (with $L C_{i}, i=1, \cdots, I$ ) available for the smart meter $S M_{j}$ to switch independently. The jamming probability $\operatorname{Prob}\left(S M_{j}\right)^{L C S-C H}$ for $S M_{j}$ becomes:

$$
\begin{aligned}
& \operatorname{Prob}\left(S M_{j}\right)^{L C S-C H} \\
= & \sum_{i=1}^{I} \operatorname{Prob}\left(f^{J}(t)=f_{k} \& f^{S M_{j}}(t)=f_{k} \mid S N R_{L C_{i}, S M_{j}}^{k}<\gamma_{0}\right) \\
& \times \operatorname{Prob}\left(S N R_{L C_{i}, S M_{j}}^{k}<\gamma_{0} \mid L C_{i}\right) \times \operatorname{Prob}\left(L C_{i}\right)
\end{aligned}
$$

The first term in equation 8 represents the jamming probability for a single local controller, which is the same as equation 7 . In addition, the probability for a particular smart meter switching among $I$ local controllers can be represented as $\operatorname{Prob}\left(L C_{i}\right)=\frac{1}{I}$. Therefore, we can further derive as follows: 


$$
\begin{aligned}
& \operatorname{Prob}\left(S M_{j}\right)^{L C S-C H} \\
= & \sum_{i=1}^{I} \frac{1}{n \times N_{i}} \operatorname{Prob}\left(S N R_{L C_{i}, S M_{j}}^{k}<\gamma_{0}\right) \times \frac{1}{I} \\
= & \frac{1}{I \times n} \sum_{i=1}^{I}\left(\frac{1}{N_{i}} \int_{-\infty}^{\gamma_{0}} \frac{1}{2 \sigma \sqrt{\pi}} e^{-\frac{\left(S N R_{L C_{i}, S M_{j}}^{k}-\mu\right)^{2}}{4 \sigma^{2}}}\right) \\
< & \operatorname{Prob}\left(S M_{j}\right)^{C H} .
\end{aligned}
$$

Therefore, the jamming probability of a smart meter under the LCS-CH scheme is lower than that under the $\mathrm{CH}$ scheme. And smart meters have higher possibility to deliver the data successfully to local controllers.

\subsection{Impact of Jamming Power and Shadowing Effect on Jamming Probability}

In this subsection, we discuss the impact of the jamming power and the shadowing channel on the jamming probability of the communication between LC and SM. Increasing jamming power typically causes more severe interference at $\mathrm{LC}$ which will result in higher jamming probability. However, how the shadowing factor, which represents the variations of the wireless channel, affects the jamming probability depends on both the jammer's transmission power and the SNR threshold. We are thus interested in the jamming probability under the impact of jamming power and shadowing. The following theoretical analysis focuses on one pair of LC and SM with the presence of single jammer. We provide the statistical results on the jamming probability involving multiple pairs of LCs and SMs in later section.

Assuming $\lambda$ is the SNR threshold for jamming detection for the channel between LC and SM, the jamming probability can be represented as:

$$
\begin{aligned}
\operatorname{Prob}_{J} & =1-\operatorname{Prob}\left(P_{R}>\lambda\right) \\
& =1-\int_{P_{R}=\lambda}^{+\infty} \frac{1}{\sigma_{R} \sqrt{2 \pi}} e^{-\frac{\left(P_{R}-\mu_{R}\right)^{2}}{2 \sigma_{R}^{2}}} d P_{R} \\
& =\int_{-\infty}^{P_{R}=\lambda} \frac{1}{\sigma_{R} \sqrt{2 \pi}} e^{-\frac{\left(P_{R}-\mu_{R}\right)^{2}}{2 \sigma_{R}^{2}}} d P_{R}
\end{aligned}
$$

where $P_{R}=\left(P_{T}-P_{J}\right) \sim \operatorname{Gauss}\left(\mu_{R}, \sigma_{R}^{2}\right), \mu_{R}=\overline{P_{T}}-\overline{P_{J}}$. $P_{R}$ is the SNR at LC, $P_{T}$ is the received transmission power from $\mathrm{SM}$, and $P_{J}$ is the received transmission power from jammer.

1. We first study the jamming probability affected by jammer transmission power. Given two different jammer transmission power $P_{J}$ and $P_{J}+\Delta P_{J}$, where $\Delta P_{J}>0$, the expected received jamming power are $\mu_{R}$ and $\mu_{R}-\Delta P_{J}$ respectively. We assume the jamming probabilities for the two different received jamming power are $\operatorname{Prob}_{J}$ and $\operatorname{Prob}_{J}^{\prime}$ with fixed shadowing factor $\sigma_{R}$ and SNR threshold $\lambda$. Then we have:

$$
\begin{aligned}
\operatorname{Prob}_{J}-\operatorname{Prob}_{J}^{\prime} & =\int_{-\infty}^{\lambda} \frac{1}{\sigma_{R} \sqrt{2 \pi}} e^{-\frac{\left(P_{R}-\mu_{R}\right)^{2}}{2 \sigma_{R}^{2}}} d P_{R} \\
& -\int_{-\infty}^{\lambda} \frac{1}{\sigma_{R} \sqrt{2 \pi}} e^{-\frac{\left(P_{R}-\left(\mu_{R}-\Delta P_{J}\right)\right)^{2}}{2 \sigma_{R}^{2}}} d P_{R} \\
& =\phi\left(\frac{\lambda-\left(\mu_{R}-\Delta P_{J}\right)}{\sigma_{R}}\right)-\phi\left(\frac{\lambda-\mu_{R}}{\sigma_{R}}\right) .
\end{aligned}
$$

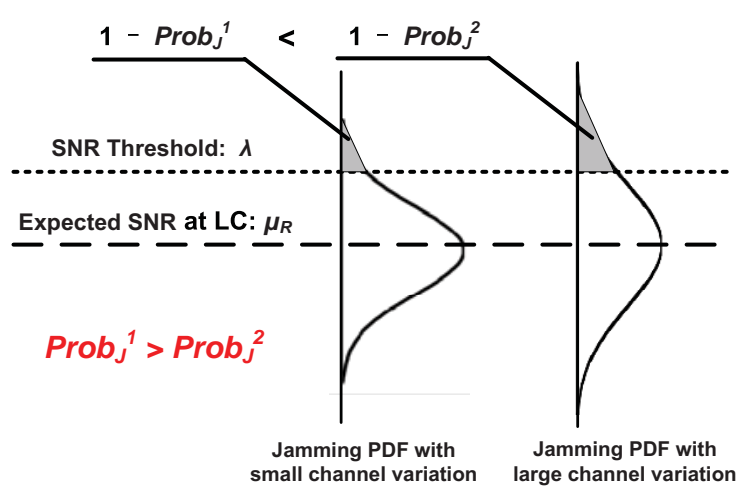

Fig. 5. Illustration of jamming probability on the wireless channel with different shadowing factors.

According to the property of Gaussian distribution, it is straightforward to find that when $P_{J}$ increases, the jamming probability $\operatorname{Prob}_{J}$ also increases.

$$
\forall \lambda, \Delta P_{J}>0, \sigma_{R} \rightarrow \Rightarrow \operatorname{Prob}_{J} \uparrow
$$

where $\rightarrow$ represents the value keeps constant, and $\uparrow$ represents the value is increasing.

2. We next analyze how the shadowing factor affects the jamming probability. When the shadowing factor $\sigma_{R}^{2}$ increases, the jamming probability is not a monotone function, which can be illustrated from the example shown in Figure 5. With a fixed received jamming power, when the SNR threshold is larger than the ratio between the received transmission power from jammer and SM, the jamming probability $\operatorname{Prob}_{J}^{1}$ for the wireless channel with small variation is higher than the probability $\operatorname{Prob}_{J}^{2}$ for the channel with larger variation. Theoretically, the change of jamming probability with respect to shadowing factor is summarized as follows:

$$
\left\{\begin{array}{l}
\lambda>\mu_{R}, \Delta P_{J}=0, \sigma_{R} \uparrow \Rightarrow \operatorname{Prob}_{J}=1-\phi\left(\frac{\lambda-\mu_{R}}{\sigma_{R}}\right) \downarrow \\
\lambda=\mu_{R}, \Delta P_{J}=0, \sigma_{R} \uparrow \Rightarrow \operatorname{Prob}_{J}=1-\phi(0) \rightarrow \\
\lambda<\mu_{R}, \Delta P_{J}=0, \sigma_{R} \uparrow \Rightarrow \operatorname{Prob}_{J}=1-\phi\left(\frac{\lambda-\mu_{R}}{\sigma_{R}}\right) \uparrow
\end{array}\right.
$$

where $\downarrow$ represents the value is decreasing.

3. Integrating the impact of the jamming power and the shadowing factor, we find that the jamming probability is not a monotone function with respect to the channel variation while jammer transmission power is increasing. Particularly, when $\lambda \leq \mu_{R}$ and $\Delta P_{J}>0$, it is straightforward to derive from Equation 12 and 13 that the jamming probability $\operatorname{Prob}_{J}$ increases when increasing the channel variation:

$$
\lambda \leq \mu_{R}, \Delta P_{J}>0, \sigma_{R} \uparrow \Rightarrow \operatorname{Prob}_{J} \uparrow .
$$

When $\lambda>\mu_{R}$ and $\Delta P_{J}>0$, in order to analyze the change of jamming probability, we seek to determine such a relationship $\Delta P_{J}=f\left(\Delta \sigma_{R}\right)$ between increased jamming power $\Delta P_{J}$ and increased shadowing factor $\Delta \sigma_{R}$ that would result in constant jamming probability. For $\Delta P_{J}>f\left(\Delta \sigma_{R}\right)$ or $\Delta P_{J}<f\left(\Delta \sigma_{R}\right)$, the jamming probability follows different trends. Given two pairs of received jamming power and shadowing factor, i.e., $\left[P_{R}, \sigma_{R}\right]$ and $\left[P_{R}+\Delta P_{J}, \sigma_{R}^{\prime}=\sigma_{R}+\Delta \sigma_{R}\right]$, where $\sigma_{R}$ and $\sigma_{R}^{\prime}$ are two different shadowing factors and $\Delta \sigma_{R}>0$, the resulted jamming probabilities are $\operatorname{Prob}_{J}$ and $\operatorname{Prob}_{J}^{\prime}$ satisfying the following condition: 


$$
\begin{aligned}
\operatorname{Prob}_{J} & =\operatorname{Prob}_{J}^{\prime} \\
1-\operatorname{Prob}\left(P_{R}>\lambda\right) & =1-\operatorname{Prob}^{\prime}\left(P_{R}>\lambda\right)
\end{aligned}
$$

We then expand the above equation and obtain the relationship between increased jammer transmission power $\Delta P_{J}$ and the two shadowing factors $\sigma_{R}$ and $\sigma_{R}^{\prime}$ as follows:

$$
\begin{aligned}
& \int_{P_{R}=\lambda}^{+\infty} \frac{1}{\sigma_{R} \sqrt{2 \Pi}} e^{-\frac{\left(P_{R}-\mu_{R}\right)^{2}}{2 \sigma_{R}^{2}}} d P_{R}= \\
& \int_{P_{R}=\lambda}^{+\infty} \frac{1}{\sigma_{R}^{\prime} \sqrt{2 \Pi}} e^{-\frac{\left(P_{R}-\left(\mu_{R}-\Delta P_{J}\right)\right)^{2}}{2\left(\sigma_{R}^{\prime}\right)^{2}}} d P_{R} \\
\Rightarrow & \phi\left(\frac{\lambda-\mu_{R}}{\sigma_{R}}\right)=\phi\left(\frac{\lambda-\left(\mu_{R}-\Delta P_{J}\right)}{\sigma_{R}^{\prime}}\right) \\
\Rightarrow & \frac{\lambda-\mu_{R}}{\sigma_{R}}=\frac{\lambda-\left(\mu_{R}-\Delta P_{J}\right)}{\sigma_{R}^{\prime}} \\
\Rightarrow & \lambda \sigma_{R}^{\prime}-\mu_{R} \sigma_{R}^{\prime}=\lambda \sigma_{R}-\mu_{R} \sigma_{R}+\Delta P_{J} \sigma_{R} \\
\Rightarrow & \Delta P_{J}=\frac{\left(\lambda-\mu_{R}\right)\left(\sigma_{R}^{\prime}-\sigma_{R}\right)}{\sigma_{R}} \\
= & \frac{\left(\lambda-\mu_{R}\right)}{\sigma_{R}} \Delta \sigma_{R}=f\left(\Delta \sigma_{R}\right) .
\end{aligned}
$$

Given the relationship $\Delta P_{J}=\frac{\left(\lambda-\mu_{R}\right)}{\sigma_{R}} \Delta \sigma_{R}$ and the SNR threshold $\lambda>\mu_{R}$, from the above analysis we find that the jamming probability keeps constant. Further, it is also straightforward to obtain that $\operatorname{Prob}_{J}$ decreases when $\Delta P_{J}>\frac{\left(\lambda-\mu_{R}\right)}{\sigma_{R}} \Delta \sigma_{R}$, while increases when $\Delta P_{J}<\frac{\left(\lambda-\mu_{R}\right)}{\sigma_{R}} \Delta \sigma_{R}$. Therefore, when both the jamming power and the shadowing factor vary we can conclude that the jamming probability will present the following trend:

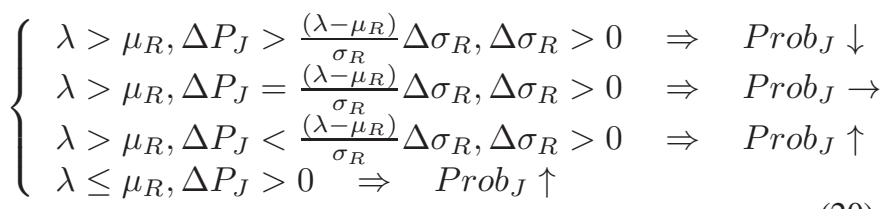

The above analysis shows that the jamming probability does not follow a monotonic changing trend when changing the jamming power or shadowing factor.

Given a smart grid network including multiple LCs distributed in a wide area, which have different distances from the jammer, it would result in different received jamming power at different LCs. Further, as the shadowing factor is dominated by different wireless environment and channel condition, it varies under different environments. For example, the downtown or residential area suffers from severe multi-path effects due to many highrise buildings, which leads to large channel variation; whereas the suburban area experiences less multi-path effects, resulting in small channel variation.

Integrating the impact of jamming power and shadowing factor, the jamming probability would follow an irregular pattern for a smart grid network. Since the received jamming power is governed by the distances from jammers and shadowing factors, the jamming probability study above thus provides insightful information on identifying useful features of jammers including jamming power and hopping patterns and understanding how environmental factors (i.e. shadowing effects) would affect the efficiency of jamming. In Section 7.4, we evaluate the jamming probability under the impact from jamming power and shadowing factor for a specific simulated network distribution.

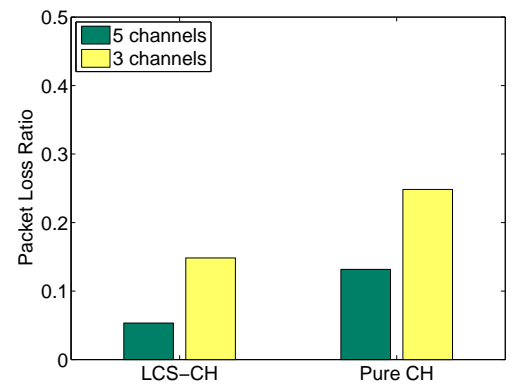

Fig. 6. Experimental Evaluation of LCS-CH in ZigBee Network

\section{ImPLEMENTATION OF CONTROLLER SWITCHING SCHEME IN ZigBEE NeTWORK}

The smart communication subsystem for smart meters and local controllers is usually deployed using a ZigBee network [1]. It is thus essential to show the feasibility of applying the proposed local controller switching scheme in the ZigBee network besides providing theoretical analysis for our framework in Section 5. We build a testbed using MicaZ motes that implement our local controller switching scheme and evaluate its performance when a jammer is present. MicaZ sensor nodes have a $2.4-2.48 \mathrm{GHz}$ Chipcon CC2420 Radio and communicate using the ZigBee protocol.

\subsection{Testbed Setup}

Our testbed consists of 6 motes with 4 acting as smart meters $\left(S M_{j}, j=1, \cdots, 4\right)$ and 2 as local controllers $\left(L C_{i}, i=\right.$ $1, \cdots, 2)$, and a 7 th mote deployed as a jammer. The two local controllers can forward the collected data from smart meters to the substation, which is represented by a mote base-station. Each smart meter communicates to one primary local controller with $S M_{2}$ and $S M_{3}$ covered by both local controllers. During our experiments, the jammer transmits with a higher transmission power $(7 \mathrm{dBm})$ than smart meters $(5 \mathrm{dBm})$. Two testing scenarios with each local controller having 3 and 5 available channels respectively are conducted.

\subsection{Implementation and Results}

We implement LCS-CH on motes and compare it with pure channel hopping technique. We emulate two operating scenarios in the smart grid under jamming: (1) smart meters communicate with their primary local controllers using a predefined channel hopping sequence; and (2) smart meters actively switch between local controllers using their respective channel hopping sequences. During testing, we allow the system to operate using pure channel hopping and LCS-CH schemes for 5 minutes each with a packet sending rate from the smart meter set at $4 p k t / s e c$. We then examine the packet loss ratio at the substation. The results are presented in figure 6. We observe that our proposed LCS-CH scheme significantly outperforms pure channel hopping scheme with much lower packet loss ratio under jamming with over $40 \%$ and $60 \%$ improvement for 3 and 5 channel cases respectively. This small-scale testbed study confirms the feasibility of implementing local controller switching technique in the ZigBee network.

\section{Simulation Evaluation}

In this section, we evaluate the effectiveness of our LCS-CH scheme under different types and different numbers of jammers through a simulated smart grid communication subsystem. 


\subsection{Simulation Setup}

The smart grid communication system is simulated using Matlab 2013b running on the desktop with Intel i7 CPU and 4G memory. Our simulated smart grid communication subsystem is a $500 m \times 500 m$ square area with 200 smart meters and 40 or 60 local controllers randomly placed. Each smart meter is associated with its closest local controller as its primary local controller and can transmit at $4 \mathrm{pkt} / \mathrm{sec}$ when accessing to the wireless channel. To simulate the wireless channel, we adopt the log-normal shadowing model for signal propagation and the parameters are set following a typical outdoor environment modeled by many previous works [31]-[34]: $P L_{0}=4, \gamma=0.6, d_{0}=5$ and $X_{g}$ is the shadow fading which follows the zero mean Gaussian distribution with the variance varying from 0 to $3 \mathrm{dBm}^{2}$. The default transmission power of jammers is $20 \mathrm{dBm}$, while it is $17 \mathrm{dBm}$ for smart meters. The SNR threshold is set to $3 d B$ for jamming detection.

The simulation is conducted as follows: All smart meters are ready to transmit when the simulation starts while the jammer also starts to hop among available channels in an attempt to disrupt the communications between smart meters and their local controllers. Each local controller is assigned with 5 channels. The smart meters are following the channel hopping sequence defined by the proposed LCS-CH framework to communicate with neighboring local controllers including the primary local controller, while the jammer randomly hops among the channels associated with the target local controller. Particularly, we set the jammer hopping rate as 12channel/sec, which is three times that of a smart meter's hopping rate (i.e., 4channel/sec). The collision occurs when smart meter and jammer hop to the same channel. In our simulation, we consider varying number (either one or multiple) of random and reactive jammers which are randomly placed in the simulation area. For multiple jammers, we study both independent and colluded jammers and use two jammers as a representative example. We ran the simulation for each scenario 10, 000 times to obtain relevant statistical results.

\subsection{Metrics}

We define Jammed Slot Ratio (JSR) to evaluate the effectiveness of our proposed LCS-CH scheme. We first define $\kappa_{i}(t)$ as the status (i.e., jammed or not jammed) at the smart meter $S M_{i}$ during time slot $t$ :

$$
\begin{array}{ll}
\kappa_{i}(t)=1 & \text { jammed } \\
\kappa_{i}(t)=0 & \text { not jammed } .
\end{array}
$$

We further use $\kappa_{i}^{s}(t)$ to represent the status of the smart meter $S M_{i}$ at time slot $t$ when our proposed LCS-CH scheme (i.e., with local controller switching) is applied.

The JSR is then defined as the ratio between the number of jammed time slots to the number of un-jammed ones of the smart meter under jamming is present.

Jammed Slot Ratio (JSR). When LCS-CH is applied, the JSR is represented as:

$$
J S R^{s}=\frac{\sum_{t=1}^{T} \sum_{i=1}^{M} \kappa_{i}^{s}(t)}{M \times T},
$$

where $T$ is the total number of time slots under study and $M$ is the number of smart meters. Similarly, when only the channel hopping $(\mathrm{CH})$ technique is applied, the JSR becomes:

$$
J S R=\frac{\sum_{t=1}^{T} \sum_{i=1}^{M} \kappa_{i}(t)}{M \times T} .
$$

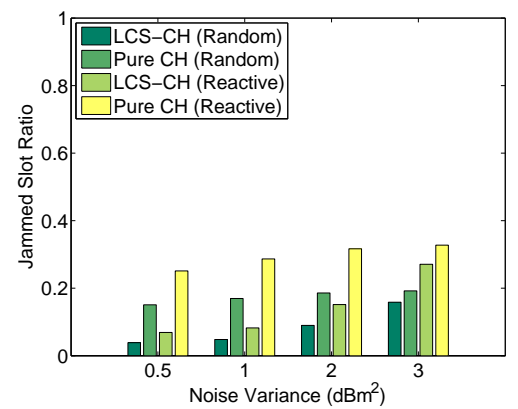

(a) $40 \mathrm{LCs}$

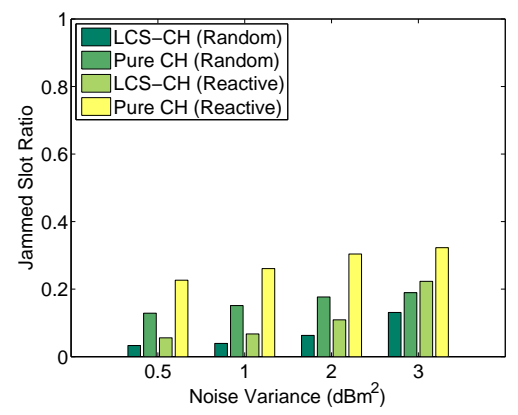

(b) $60 \mathrm{LCs}$

Fig. 7. Single jammer case: Comparison of Jammed Slot Ratio (JSR) between LCS-CH and Pure $\mathrm{CH}$.

Improvement Percentage $(\eta)$. We further define the JSR improvement percentage, which represents the percentage of jamming slot ratio reduced under the LCS-CH scheme when compared with the pure channel hopping scheme, as:

$$
\eta=\frac{J S R-J S R^{s}}{J S R} .
$$

\subsection{Results}

\subsubsection{Single Jammer case}

We first study the performance of our proposed framework when a single jammer is present. Figure 7 (a) and (b) depict the JSR comparison between the proposed LCS-CH scheme and pure frequency hopping (i.e., Pure FH) scheme under both random and reactive jammers when the variance of shadowing is varied from $0 \mathrm{dBm} \mathrm{m}^{2}$ to $3 \mathrm{dBm}^{2}$ with 40 and 60 local controllers, respectively. We observe that the JSR of the LCS-CH scheme is substantially less than that of the pure FH scheme under both 40 and 60 local controllers settings. This observation indicates that the proposed scheme has a much lower jammed slot ratio, and thus has significantly performance improvement over the Pure FH scheme. Specifically, JSR drops from $17.1 \%(15.1 \%)$ to $4.8 \%(3.9 \%)$ with 40 (60) local controllers when the variance of shadowing is $1 \mathrm{dBm} \mathrm{m}^{2}$ under random jamming. Similarly, for the reactive jammer, JSR drops from $29 \%(26 \%)$ to $8.3 \%$ (6.7\%) with 40 (60) local controllers when the variance of shadowing is $1 \mathrm{dBm}^{2}$. This is because the proposed LCS-CH scheme provides more flexibility on channel hopping among multiple local controllers. It is thus harder for a jammer to disrupt the communication between smart meters and local controllers. We also find that the JSR of the proposed scheme under 60 local controllers is smaller than that of under 40 local controllers, indicating each smart meter having more choices for channel switching when more local controllers are deployed. 


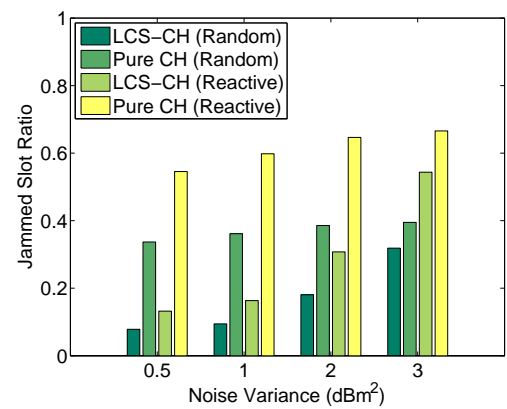

(a) Independent jammers

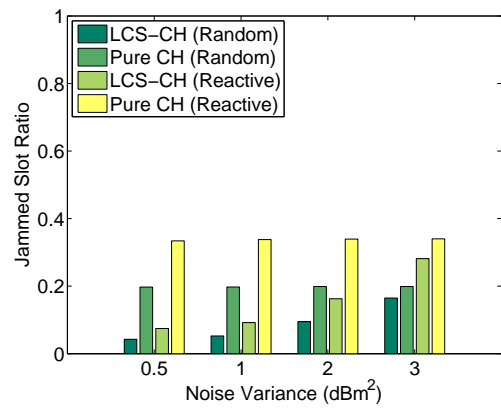

(b) Colluded jammers

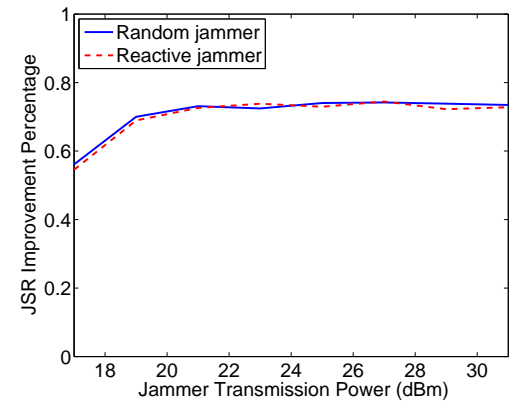

(a) $40 \mathrm{LCs}$

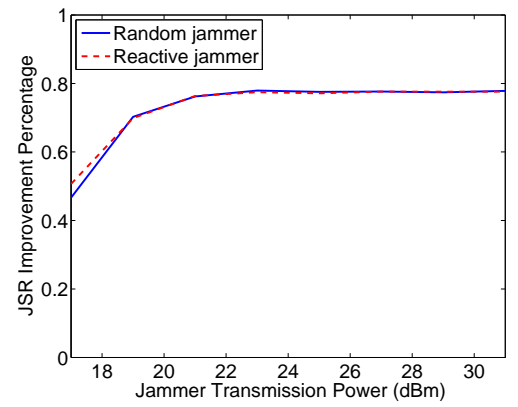

(b) $60 \mathrm{LCs}$
Fig. 8. Two jammers case: Comparison of JSR between LCS-CH and Pure $\mathrm{CH}$ with 40 local controllers.

Furthermore, we observe that the JSR is increasing as the noise power (i.e., variance of shadowing) increases. This is because a higher noise power results in a lower signal-to-noise ratio, which affects the communication between local controllers and smart meters even in normal conditions. This causes the decreasing of the number of local controllers that a smart meter can communicate with, especially those which are located relatively farther away from the smart meter. When the noise power is large enough (e.g., larger than $3 \mathrm{dBm}^{2}$ ), the smart meter could only maintain the communication with its primary local controller (assuming the primary local controller is the closest controller to the smart meter). This will make the JSR under the LCS-CH scheme approaching to that of Pure FH scheme. But still, the performance of LCS-CH is better than that of Pure FH scheme.

Additionally, we find that the reactive jammer is more harmful than the random jammer. Once the reactive jammer captures one active channel, it could disrupt all the packets transmitted during the whole time slot. This is different from a random jammer, who only disrupts the communication in a portion of one time slot due to the fast hopping rate of jammers. Therefore, the JSR under a reactive jammer is higher than that of a random jammer.

\subsubsection{Multiple Independent Jammers case}

We next examine how our framework reacts when there are multiple independent jammers present in the smart grid communication subsystem. Figure 8(a) presents the JSR comparison of the proposed LCS-CH scheme and pure FH scheme when two jammers are present with 40 local controllers. We observe that the JSR of the LCS-CH scheme is significantly lower than that of the pure $\mathrm{FH}$ scheme for all studied cases using random and reactive jammers respectively. As expected, when compared to the single jammer case, the JSR of pure FH scheme increases sharply under two jammers case due to more channels are affected by multiple jammers. The JSR of our proposed LCS-CH under two jammers is about twice of that under a single jammer case.
Fig. 9. The JSR improvement percentage for single jammer under different jammer transmission power with 40 and 60 local controllers, respectively.

This is because having two jammers independently disrupt the channels on a local controller results in similar performance as the summation of JSRs from two independent jamming scenarios with a single jammer. The performance under 60 local controllers exhibits better performance than the 40 local controllers case but was omitted due to space limitation.

\subsubsection{Multiple Colluded Jammers Case}

We further examine the case with multiple colluded jammers in the smart grid communication subsystem. The JSR comparison of the proposed LCS-CH scheme and pure FH scheme under two colluded jammers with 40 local controllers are presented in figure 8 (b). The performance under 60 local controllers is again omitted due to space limitation. We find that the JSR of our proposed LCS-CH is much better than that of pure FH. When compared to the JSR under a single jammer, we observe that the JSR of LCS-CH under two colluded jammers increases about only $0.5 \%$, which indicates that colluded jammers have accumulated impact on the channels between smart meters and local controllers. Since the two jammers are randomly distributed in the testing area, the accumulated impact is not that obvious compared with a single jammer case. It also shows the robustness of our proposed LCS-CH scheme when dealing with colluded jammers. Further, we observe that having two colluded jammers is less harmful than having two independent jammers for both LCS-CH and Pure FH schemes from our simulation results.

\subsubsection{Impact of Jamming Power}

Finally, we study how our proposed framework behaves when the jammer's transmission power increases. We vary the jammer's transmission power from $17 \mathrm{dBm}$ to $30 \mathrm{dBm}$, while maintaining the transmission power of smart meters at $17 \mathrm{dBm}$ with constant noise power level set at $1 \mathrm{dBm}^{2}$. Figure 9 depicts the JSR improvement percentage of LCS-CH over Pure FH with both a 


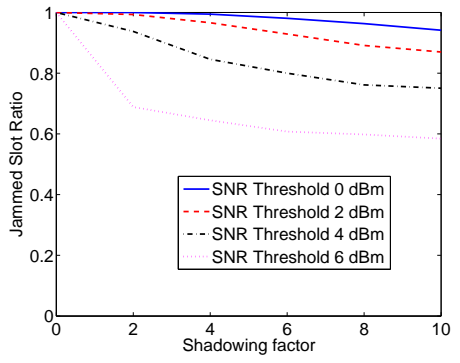

(a) Jamming Power $10 \mathrm{dBm}$

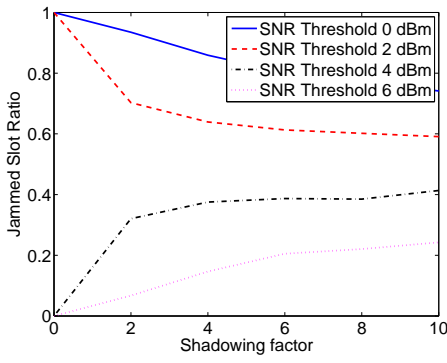

(b) Jamming Power $14 \mathrm{dBm}$

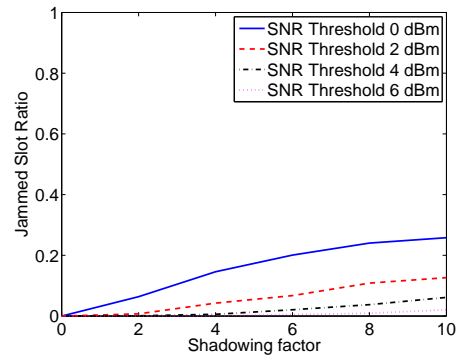

(c) Jamming Power $20 \mathrm{dBm}$

Fig. 10. JSR under the impact of jamming power and shadowing factor for single pair of LC and SM.

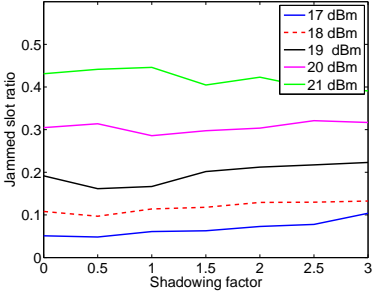

(a) Pure channel hopping, random

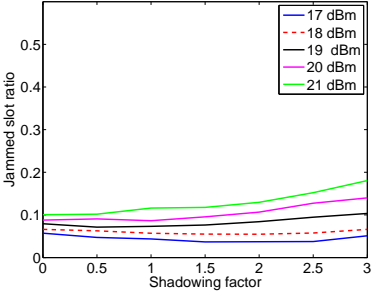

(b) LCS-CH, random

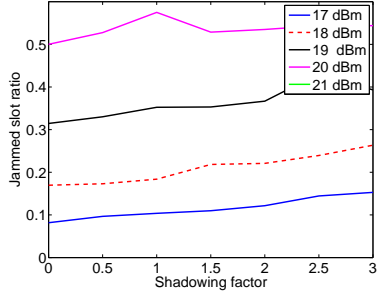

(c) Pure channel hopping, reactive

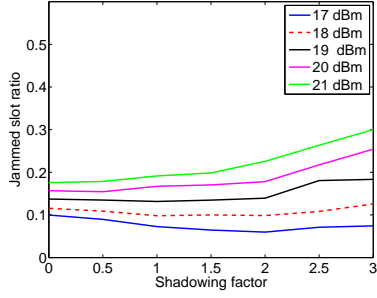

(d) LCS-CH, reactive

Fig. 11. Average JSR under the impact of jammer transmission power and shadowing factor across the network.

single random and reactive jammer cases respectively the transmission power of the jammer is varied. We observe that our LCS$\mathrm{CH}$ achieves large JSR improvement (over 50\%) under different number of local controllers for both random and reactive jammers. This is very encouraging as it indicates our framework is highly effective when the adversary increases the jammer's transmission power. The JSR improvement becomes stable beyond $22 \mathrm{dBm}$ of jammer transmission power. This is because the jammers with low transmission power have limited impact on the signal-tonoise ratio of the communication links between smart meters and local controllers. They can mostly affect the communication links between a smart meter and far away controllers. When the jamming power increases, more communication links will get affected. Once the transmission power of jammer becomes large enough, the communication links between the smart meter and all the local controllers will get affected resulting in low SNR if they are on the same channel as the jammer. As the jamming power increases, the jamming capability becomes saturated.

\subsubsection{Impact of Jammer Transmission Power and Shadow- ing Factor}

We first study the jamming probability for a single pair of SM and LC when varying jammer's transmission power and shadowing factor. Given the SM transmission power at $17 \mathrm{dBm}$, and the jammer transmission powers fixed at $10 \mathrm{dBm}, 14 \mathrm{dBm}$ and $20 \mathrm{dBm}$ respectively, we examine the jamming slot ratio when the shadowing factor varies from $0 \mathrm{dBm}$ to $10 \mathrm{dBm}$. As shown in Figure 10, the jamming slot ratio has different increasing or decreasing trends as the shadowing factor is increased with different jamming powers. This observation matches our theoretical analysis presented in Section 5. Particularly, the jammed slot ratio decreases when increasing the shadowing factor with the jammer transmission power of $10 \mathrm{dBm}$, whereas the jammed slot ratio increases when increasing the shadowing factor under the jammer transmission power of $20 \mathrm{dBm}$. When jammer transmission power is $14 \mathrm{dBm}$, the changes of the jammed slot ratio when increasing the shadowing is related to the SNR threshold. In particular, the jammed slot ratio decreases under the SNR threshold $0 \mathrm{dBm}$ and $2 d B m$, while it increases under the SNR threshold $4 d B m$ and $6 d B m$.

We next study the impact of jammer transmission power and shadowing factor on the jamming probability in a simulated smart grid network, which consists of 60 LCs and 200 SMs randomly distributed in a $500 m \times 500 m$ area. We calculate the average jamming slot ratio across the whole network, where the jamming power changes from $17 \mathrm{dBm}$ to $21 \mathrm{dBm}$, and the shadowing factor varies from $0.5 \mathrm{dBm}$ to $3 \mathrm{dBm}$. Given the fixed jammer transmission power, we observe that the average jammed slot ratio does not monotonically increase or decrease with respect to shadowing factor, which is shown in Figure 11. Particularly, for the proposed LCS-CH scheme, the jammed slot ratio decreases first and then increases as the shadowing factor increases under the lower jammer transmission powers (i.e., $17 \mathrm{dBm}$ and $18 \mathrm{dBm}$ ), while it keeps increasing with higher transmission powers (i.e., $19 \mathrm{dBm}, 20 \mathrm{dBm}$ and $21 \mathrm{dBm}$ ). Furthermore, the jammed slot ratio of the pure channel hopping scheme is always higher than that of the proposed LCS-CH scheme, which demonstrates the effectiveness of our proposed scheme under different wireless environments.

\subsubsection{Throughput and Communication Overhead Study with Retransmission Scheme}

In this subsection, we first study the throughput under the proposed framework after integrating with the retransmission scheme. The throughput reflects the efficiency of the proposed framework, and is defined as the average number of packets successively delivered and acknowledged at LC per time slot $(p k t / T)$, where $T$ represents unit time slot. Note that each time slot only allows to transmit one packet, and every 600 time slots of packets transmission is followed by 30 time slots of acknowledgment for successful packet delivery by LCs. 


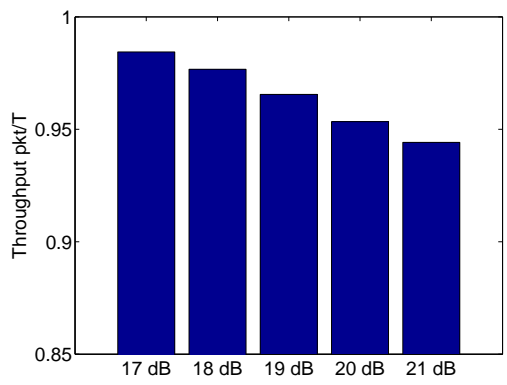

(a) Random Jammer

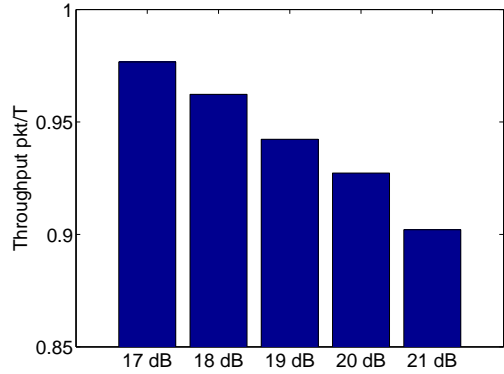

(b) Reactive Jammer

Fig. 12. Throughput under different jammer transmission powers when applying the retransmission scheme.

Figure 12 shows the channel throughput under different jammer transmission powers for both random and reactive jammers. Particularly, as the jammer transmission power increases from 17 $\mathrm{dBm}$ to $21 \mathrm{dBm}$, the throughput always maintains at high level (i.e., above $0.95 p k t / T$ and $0.9 p k t / T$ for random and reactive jammer respectively), which indicates that the proposed LCS-CH framework is effective in defending against jamming attacks.

We also study the communication overhead incurred by the proposed retransmission scheme. The communication overhead is defined as the relative ratio between the number of retransmitted data packets and the total number of data packets transmitted by smart meters. Following the time slot arrangement in throughput study, the communication overhead varying with the jammer transmission power are presented in Figure 13. Specifically, the communication overhead is as low as $2.8 \%$ and $5.2 \%$ for random and reactive jammer respectively even when the transmission power of jammer goes up to $21 \mathrm{dBm}$. Such low communication overhead confirms that the proposed retransmission scheme does not incur high communication overhead.

\section{Optimization of local Controller Place- MENT}

In Section 5.2, we study the jamming probability with fixed distances between jammer and LCs \& SMs by varying the jammer transmission power and shadowing factor. In contrast, in this section we consider another aspect of the proposed framework. Assuming the jammer transmission power and shadowing factor are fixed, we explore the placement of Local controllers to get maximized overlapping coverage of smart meters. Generally, the deployment of smart meters in a geographical area is usually fixed. Given the total number of local controllers planned in this geographical area, it is useful to perform the deployment in such a way that each smart meter can communicate with the maximum number of nearby controllers to facilitate active local controller switching under jamming. To address this challenge in the selfhealing smart grid, our framework proposes the optimal placement

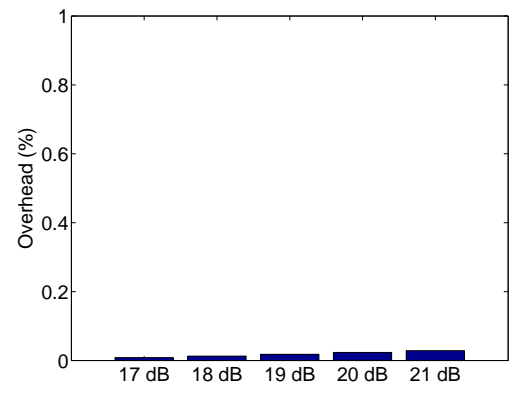

(a) Random Jammer

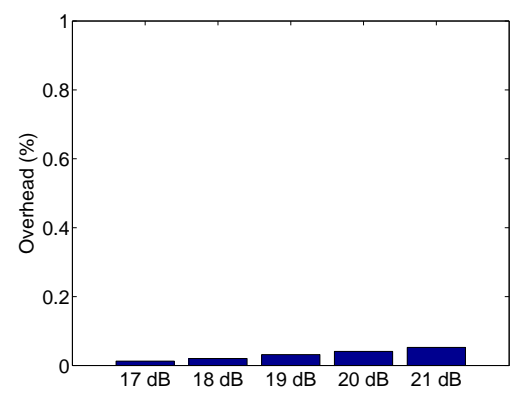

(b) Reactive Jammer

Fig. 13. Communication Overhead under different jammer transmission powers when applying the retransmission scheme.

of a fixed number of local controllers to maximize the overlapping coverage of each smart meter.

Assume there are $M$ smart meters and $I$ local controllers in a specific geographic region. We formulate the smart grid communication subsystem network in this region into a connected, undirected graph, which is represented by a neighborhood adjacency matrix $C_{I \times M}$ between smart meter and local controller as follows:

$$
C_{I \times M}=\left[\begin{array}{cccc}
l_{1,1} & l_{1,2} & \cdots & l_{1, M} \\
l_{2,1} & l_{2,2} & \cdots & l_{2, M} \\
& \cdots & \cdots & \\
l_{I, 1} & l_{I, 2} & \cdots & l_{I, M}
\end{array}\right]
$$

where each element of the graph $l_{i, j}$ (with $i=1, \cdots, I$ and $j=1, \cdots, M$ ) represents a communication link between a local controller $L C_{i}$ and a smart meter $S M_{j}$ under normal operations. When a smart meter $S M_{j}$ can communicate with a local controller $L C_{i}$, the corresponding element $l_{i, j}$ in the matrix $C_{I \times M}$ is 1 , otherwise it is 0 .

Whether a smart meter $S M_{j}$ is covered or not by a local controller $L C_{i}$ depends on the signal propagation model and the distance between them. The received power at the local controller $L C_{i}$ should exceed the predefined threshold $\gamma_{0}$, which guarantees successful packet delivery. Therefore, the communication link $l_{i, j}$ should satisfy the following condition:

$$
\begin{gathered}
l_{i, j}= \begin{cases}1 & P_{L C_{i}, S M_{j}}>\gamma_{0} ; \\
0 & \text { otherwise; }\end{cases} \\
P_{L C_{i}, S M_{j}}=P_{T}-P L_{0}-10 \gamma 1 \log _{10}\left(\frac{\left\|q_{i}^{L C}-q_{j}^{S M}\right\|}{d_{0}}\right)-X_{g},
\end{gathered}
$$

where $q_{j}^{S M}$ (with $j=1, \cdots, M$, ) and $q_{i}^{L C}$ (with $i=1, \cdots, I$,) represent the position of a smart meter $S M_{j}$ and local controller $L C_{i}$ respectively. 
Our objective is to find the optimal placement of the $I$ local controllers with positions $q_{i}^{L C}, i=1, \cdots, I$, in the network such that each smart meter can be covered by at least $\mathrm{k}$ local controllers. Therefore, the optimization problem of local controller placement can be formulated as:

$$
\begin{array}{r}
\underset{q_{i}^{L C}, i=1, \cdots, I}{\arg \max } 1_{1 \times I} C_{I \times M} 1_{M \times 1} \\
\text { s.t. } 1_{1 \times I} C_{I \times n} v_{j} \geq k
\end{array}
$$

where $1_{1 \times I}$ and $1_{M \times 1}$ are $I$-length column and $M$-length row vector with all 1 's elements. $v_{j}$ is a $M$-length column vector with only $j$ th element equals to 1 and all other elements are 0 . Note that the positions of smart meters $q_{j}^{S M}$ are known.

Equation 26 searches for the optimal positions of all local controllers, $L C_{i}$, until the summation of all the link state $l_{i, j}$ in the neighborhood adjacency matrix $C_{I \times M}$ is maximized. To avoid the optimization process from falling into a local optimal solution, we enforce that each smart meter should be covered by at least $k$ local controllers. This optimization problem of searching for the positions of local controllers can be solved using the integer programming technique [35]. The optimal placement of local controllers serves as inputs into our proposed framework to facilitate intelligent local controller switching under jamming.

\section{Conclusion}

Jamming attacks in the last mile of the smart grid aim to disrupt the data communication between smart meters and local controllers and further launch a variety of adversarial activities. In this paper, we have exploited local controller switching to provide resilience of data delivery under jamming in the distribution network. The proposed framework enables smart meters to utilize all the available channels from nearby local controllers to ensure successful data delivery. We have further integrated a retransmission scheme into our proposed LCS-CH framework to enhance the successful data delivery. Theoretical analysis shows that our proposed intelligent local controller switching with channel hopping (LCS$\mathrm{CH})$ framework reduces the jamming probability compared to the pure channel hopping approach. Additionally, we have provided theoretical insights into the jamming probability affected by the jammer's transmission power and shadowing factor. Furthermore, our testbed using MicaZ motes shows the feasibility of implementing the intelligent local controller switching scheme in a ZigBee network. And our large-scale simulation results confirm the effectiveness of our approach even when multiple jammers are present. Finally, we have provided guidelines on the optimal placement of local controllers to ensure effective switching of smart meters under jamming, leading toward a self-healing communication subsystem in the smart grid. In our future work, we may design a mechanism for negotiating dynamic channel hopping sequences.

Acknowledgements: The preliminary results of this work have been presented at IEEE CNS 2014 [36]. Yingying Chen would like to acknowledge the support of NSF grant CNS0954020 and ARO W911NF-13-1-0288. Mooi Choo Chuah would like to acknowledge the support of a startup grant from Lehigh University. The work of H. Vincent Poor was supported in part by the National Science Foundation under Grant CMMI-1435778.

\section{RefERENCES}

[1] X. Fang, S. Misra, G. Xue, and D. Yang, "Smart grid - the new and improved power grid: A survey," IEEE Communications Surveys Tutorials, 2012.
[2] "IEEE 802.16 Standard," http://www.ieee.org.

[3] T. Goodspeed, S. Bratus, R. Melgares, R. Speers, and S. W. Smith, "Apido: Tools for exploring the wireless attack surface in smart meters." in Proc. Hawaii International Conference on System Sciences, 2012.

[4] Z. Lu, W. Wang, and C. Wang, "Hiding traffic with camouflage: Minimizing message delay in the smart grid under jamming," in Proc. IEEE Conference on Computer Communications, 2012.

[5] H. Li and Z. Han, "Manipulating the electricity power market via jamming the price signaling in smart grid," in Proc. IEEE Global Communications Conference, 2011.

[6] Y. Liu, P. Ning, and M. K. Reiter, "False data injection attacks against state estimation in electric power grids," in Proc. ACM Conference on Computer and Communications Security, 2009.

[7] O. Kosut, L. Jia, R. Thomas, and L. Tong, "Malicious data attacks on smart grid state estimation: Attack strategies and countermeasures," in Proc. IEEE International Conference on Smart Grid Communications, 2010.

[8] A. Wood, J. Stankovic, and G. Zhou, "DEEJAM: Defeating EnergyEfficient Jamming in IEEE 802.15.4-based Wireless Networks," in Proc. IEEE International Conference on Sensing, Communication, and Networking, 2007.

[9] Q. Zeng, H. Li, Z. Zhang, and D. Peng, "A frequency-hopping based communication infrastructure for wireless metering in smart grid," in Proc. Annual Conference in Information Sciences and Systems, 2011.

[10] D. Liu, J. Raymer, and A. Fox, "Efficient and timely jamming detection in wireless sensor networks," in Proc. IEEE International Conference on Mobile Ad hoc and Sensor Systems, 2012.

[11] W. Xu, "On adjusting power to defend wireless networks from jamming," in Proc. Annual International Conference on Mobile and Ubiquitous Systems: Networking and Services, 2007.

[12] A. Liu, P. Ning, H. Dai, and Y. Liu, "USD-FH: Jamming-resistant wireless communication using Frequency Hopping with Uncoordinated Seed Disclosure," in Proc. IEEE International Conference on Mobile Ad hoc and Sensor Systems, 2010.

[13] W. Xu, W. Trappe, Y. Zhang, and T. Wood, "The feasibility of launching and detecting jamming attacks in wireless networks," in Proc. ACM International Symposium on Mobile Ad Hoc Networking and Computing, 2005.

[14] Y. Liu, P. Ning, H. Dai, and A. Liu, "Randomized differential dsss: Jamming-resistant wireless broadcast communication," in Proc. IEEE Conference on Computer Communications, 2010.

[15] B. DeBruhl and P. Tague, "Mitigation of periodic jamming in a spread spectrum system by adaptive filter selection," in Proc. International Symposium on Photonic and Electromagnetic Crystal Structures, 2012.

[16] M. Strasser, S. Capkun, C. Popper, and M. Cagalj, "Jamming-resistant key establishment using uncoordinated frequency hopping," in Proc. IEEE Symposium on Security and Privacy, 2008.

[17] M. Strasser, C. Pöpper, and S. Čapkun, "Efficient uncoordinated FHSS anti-jamming communication," in Proc. ACM International Symposium on Mobile Ad Hoc Networking and Computing, 2009.

[18] V. Navda, A. Bohra, S. Ganguly, R. Izmailov, and D. Rubenstein, "Using channel hopping to increase 802.11 resilience to jamming attacks," in Proc. IEEE Conference on Computer Communications, Mini-symposium, 2007.

[19] G. Noubir and G. Lin, "Low-power DoS attacks in data wireless lans and countermeasures," ACM SIGMOBILE Mobile Computing and Communications Review, vol. 7, no. 3, pp. 29-30, 2003.

[20] K. Ma, Y. Zhang, and W. Trappe, "Mobile network management and robust spatial retreats via network dynamics," in Proc. the 1st International Workshop on Resource Provisioning and Management in Sensor Networks (RPMSN), 2005.

[21] W. Xu, W. Trappe, and Y. Zhang, "Anti-jamming timing channels for wireless networks," in Proc. the first ACM conference on Wireless network security (WiSec). New York, NY, USA: ACM, 2008, pp. 203213.

[22] M. Cagalj, S. Capkun, and J. Hubaux, "Wormhole-Based Anti-Jamming Techniques in Sensor Networks," IEEE Transactions on Mobile Computing, pp. $100-114$, January 2007.

[23] W. Shen, P. Ning, X. He, H. Dai, and Y. Liu, "Mcr decoding: A mimo approach for defending against wireless jamming attacks," in Proc. IEEE Conference on Communications and Network Security: Workshop on Physical-layer Methods for Wireless Security (PhySec), 2014.

[24] H. Liu, X. Wenyuan, Y. Chen, and Z. Liu, "Localizing jammers in wireless networks," in Proc. IEEE International Conference on Pervasive Computing and Communications, 2009, pp. 1-6.

[25] Z. Liu, H. Liu, W. Xu, and Y. Chen, "Wireless jamming localization by exploiting nodes hearing ranges," in Distributed Computing in Sensor Systems, 2010, pp. 348-361.

[26] K. Pelechrinis, I. Koutsopoulos, I. Broustis, and S. V. Krishnamurthy, "Lightweight jammer localization in wireless networks: System design 
and implementation," in Proc. Global Telecommunications Conference (GlobeCome), 2009, pp. 1-6.

[27] H. Li, L. Lai, and R. Qiu, "A denial-of-service jamming game for remote state monitoring in smart grid," in Proceedings of Annual Conference in Information Sciences and Systems, 2011.

[28] Z. Lu, W. Wang, and C. Wang, "From jammer to gambler: Modeling and detection of jamming attacks against time-critical traffic," in Proc. IEEE Conference on Computer Communications, 2011.

[29] H. Su, M. Qiui, H. Chen, Z. Lu, and X. Qin, "Jamming-resilient multi-radio multi-channel multihop wireless network for smart grid," in Proc. the Seventh Annual Workshop on Cyber Security and Information Intelligence Research, New York, NY, USA, 2011, pp. 65:1-65:1.

[30] W. Wang and Z. Lu, "Cyber security in the smart grid: Survey and challenges," Computer Networks, 2006.

[31] A. Goldsmith, Wireless Communications. New York, NY, USA: Cambridge University Press, 2005.

[32] P. Di Marco, C. Fischione, F. Santucci, and K. Johansson, "Effects of Rayleigh-lognormal fading on IEEE 802.15.4 networks," in Proc. IEEE International Conference on Communications (ICC), June 2013, pp. 1666-1671.

[33] Y. Chen and A. Terzis, "On the implications of the log-normal path loss model: An efficient method to deploy and move sensor motes," in Proc. the 9th ACM Conference on Embedded Networked Sensor Systems (SenSys), 2011, pp. 26-39.

[34] P. Di Marco, C. Fischione, F. Santucci, and K. Johansson, "Modeling IEEE 802.15.4 Networks Over Fading Channels,' IEEE Transactions on Wireless Communications, vol. 13, no. 10, pp. 5366-5381, Oct 2014.

[35] S. Yang, F. Dai, M. Cardei, and J. Wu, "On multiple point coverage in wireless sensor networks," in Proc. IEEE International Conference on Mobile Ad hoc and Sensor Systems, 2005.

[36] H. Liu, Y. Chen, M. C. Chuah, and J. Yang, "Towards self-healing smart grid via intelligent local controller switching under jamming," in Proc. IEEE Conference on Communications and Network Security (CNS), 2013, pp. 127-135.

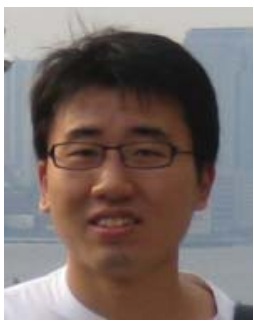

Hongbo Liu joins IUPUI as an Assistant Professor in Department of Computer Information and Graphics Technology since Aug. 2013. He received his Ph.D. degree in Electrical Engineering from Stevens Institute of Technology. His research interests include mobile and pervasive computing, cyber security and privacy and smart grid. He is the recipient of the Best Paper Award from ACM MobiCom 2011 and Best Paper Runner-up Award from IEEE CNS 2013.

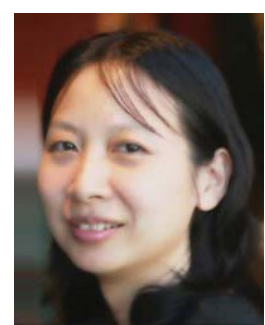

Yingying (Jennifer) Chen is an Associate Professor in the Department of Electrical and Computer Engineering at Stevens Institute of Technology. Her research interests include cyber security and privacy, mobile computing, mobile healthcare, and wireless networks. She has published extensively in these areas in both journal articles and referred conference papers. She received her Ph.D. degree in Computer Science from Rutgers University. Prior to joining Stevens, she was with Alcatel-Lucent. She is the recipient of the NSF CAREER Award and Google Research Award. She also received NJ Inventors Hall of Fame Innovator Award. She is the recipient of the Best Paper Award from ACM International Conference on Mobile Computing and Networking (MobiCom) 2011. She also received the IEEE Outstanding Contribution Award from IEEE New Jersey Coast Section each year 2005-2009. Her research has been reported in numerous media outlets.

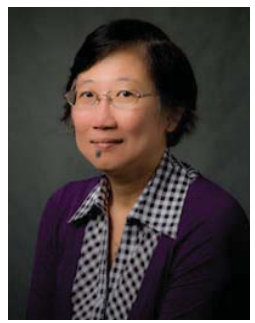

Mooi Choo Chuah is a Professor in Computer Science \& Engineering Department at Lehigh University. Her research interests include designing next generation network, mobile computing, mobile healthcare, network security, secure cyber physical systems. She received her Ph.D. degree in Electrical Engineering from University of California San Deigo. Prior to joining Lehigh, she was a Distinguished Member of Technical Staff and Technical Manager at Lucent Bell Laboratories, NJ. Based on her research work at Bell Laboratories, she has been awarded 62 US patents and 15 international patents related to mobility management, $3 G$ and next generation wireless system design, etc. She has served as a technical co-chair for IEEE INFOCOM 2010, symposium co-chair for IEEE Globecom Next Generation Networking Symposium 2013 and editor of IEEE Transaction for Mobile Computing. She is currently the Associate Editor for IEEE Transactions on Parallel \& Distributed Systems. She is also an IEEE Fellow.

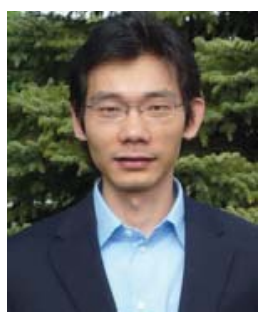

Jie Yang received his Ph.D. degree in Computer Engineering from Stevens Institute of Technology in 2011. He is currently an assistant professor in the Department of Computer Science at Florida State University. His research interests include cyber security and privacy, and mobile and pervasive computing, with an emphasis on network security, smartphone security and applications, security in cognitive radio and smart grid, location systems and vehicular applications. His research is supported by $\mathrm{Na}$ tional Science Foundation (NSF) and Army Research Office (ARO) $\mathrm{He}$ is the recipient of the Best Paper Award from IEEE Conference on Communications and Network Security (CNS) 2014 and the Best Paper Award from ACM MobiCom 2011. His research has received wide press coverage including MIT Technology Review, The Wall Street Journal, NPR, CNET News, and Yahoo News. He is a member of the IEEE.

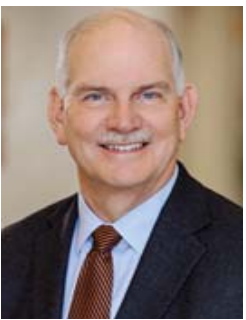

H. Vincent Poor $\left(S^{\prime} 72, M^{\prime} 77, S^{\prime} 82, F^{\prime} 87\right)$ received the Ph.D. degree in EECS from Princeton University in 1977. From 1977 until 1990, he was on the faculty of the University of Illinois at Urbana-Champaign. Since 1990 he has been on the faculty at Princeton, where he is the Michael Henry Strater University Professor of Electrical Engineering and Dean of the School of Engineering and Applied Science. Dr. Poor's research interests are in the areas of stochastic analysis, statistical signal processing, and information theory, and their applications in wireless networks and related fields such as social networks and smart grid. Among his publications in these areas is the recent book Mechanisms and Games for Dynamic Spectrum Allocation (Cambridge University Press, 2014).

Dr. Poor is a member of the National Academy of Engineering and the National Academy of Sciences, and a foreign member of Academia Europaea and the Royal Society. He is also a fellow of the American Academy of Arts and Sciences, the Royal Academy of Engineering (U. K), and the Royal Society of Edinburgh. In 1990, he served as President of the IEEE Information Theory Society, and in 2004-07 he served as the Editor-in-Chief of the IEEE Transactions on Information Theory. He received a Guggenheim Fellowship in 2002 and the IEEE Education Medal in 2005. Recent recognition of his work includes the 2014 URSI Booker Gold Medal, and honorary doctorates from Aalborg University, Aalto University, the Hong Kong University of Science and Technology and the University of Edinburgh. 